\title{
The Flavonoid Glabridin Induces OCT4 to Enhance Osteogenetic Potential in Mesenchymal Stem Cells
}

\author{
June Seok Heo, ${ }^{1,2}$ Seung Gwan Lee, ${ }^{3}$ and Hyun Ok Kim ${ }^{2,4}$ \\ ${ }^{1}$ Department of Integrated Biomedical and Life Sciences, College of Health Science, Korea University, Seoul 02841, Republic of Korea \\ ${ }^{2}$ Cell Therapy Center, Severance Hospital, Seoul 03722, Republic of Korea \\ ${ }^{3}$ Department of Health and Environmental Science, College of Health Science, Korea University, Seoul 02841, Republic of Korea \\ ${ }^{4}$ Department of Laboratory Medicine, Yonsei University College of Medicine, Seoul 03722, Republic of Korea
}

Correspondence should be addressed to Seung Gwan Lee; seunggwan@korea.ac.kr and Hyun Ok Kim; hyunok1019@yuhs.ac

Received 8 June 2017; Revised 27 September 2017; Accepted 3 October 2017; Published 14 November 2017

Academic Editor: Farid Menaa

Copyright (C) 2017 June Seok Heo et al. This is an open access article distributed under the Creative Commons Attribution License, which permits unrestricted use, distribution, and reproduction in any medium, provided the original work is properly cited.

\begin{abstract}
Mesenchymal stem cells (MSCs) are a promising tool for studying intractable diseases. Unfortunately, MSCs can easily undergo cellular senescence during in vitro expansion by losing stemness. The aim of this study was to improve the stemness and differentiation of MSCs by using glabridin, a natural flavonoid. Assessments of cell viability, cell proliferation, $\beta$-galactosidase activity, differentiation, and gene expression by reverse transcription PCR were subsequently performed in the absence or presence of glabridin. Glabridin enhanced the self-renewal capacity of MSCs, as indicated by the upregulation of the OCT4 gene. In addition, it resulted in an increase in the osteogenic differentiation potential by inducing the expression of osteogenesisrelated genes such as DLX5 and RUNX2. We confirmed that glabridin improved the osteogenesis of MSCs with a significant elevation in the expression of OSTEOCALCIN and OSTEOPONTIN genes. Taken together, these results suggest that glabridin enhances osteogenic differentiation of MSCs with induction of the OCT4 gene; thus, glabridin could be useful for stem cell-based therapies.
\end{abstract}

\section{Introduction}

Mesenchymal stem cells (MSCs) derived from various tissues are very promising sources for cellular therapies and regenerative medicine, since they are easily accessible and differentiate into a variety of cell types, including osteoblasts, chondrocytes, and adipocytes $[1,2]$. MSCs have been applied to cell-based therapies due to their numerous advantages including anti-inflammatory and immunomodulatory effects $[3,4]$. However, despite the high expansion potential of MSCs, when cultured in vitro, they easily undergo proliferation arrest prior to significant telomere shortening due to intrinsic and/or extrinsic environmental factors $[5,6]$. It is known that oxidative stress-due to an imbalance between the production of free radicals and the ability of the body to detoxify their harmful effects-is one of the main factors that induce senescence [7]. Senescence represents an arrested state in which cells remain viable but not stimulated to proliferate by serum or passage in culture. Cellular senescence of MSCs reduces functionality, which might impair their regenerative potential [8]. Therefore, MSCs need to be expanded for clinical application through in vitro long-term cultivation without early growth stop or cellular senescence.

Glabridin is an isoflavan compound found in the root extract of licorice [9]. A number of studies have reported that glabridin exhibits protective functions against oxidative stresses and cytotoxicity $[10,11]$. In addition, it has been reported to inhibit cancer stem cell-like properties of human breast cancer cells, suggesting that it could enhance the effectiveness of breast cancer therapy [12]. Recently, it was also shown that glabridin attenuates oxidative damage and cellular dysfunction and upregulates osteoblast differentiation genes in osteoblastic cells [13]. These findings suggest the interesting possibility that glabridin could have positive effects on MSC culture in vitro. Although there is some evidence that glabridin protects cells from oxidative stress, no study has investigated whether glabridin can prevent MSC senescence. We therefore hypothesized that glabridin would 
TABle 1: Primer sequences used for RT-PCR.

\begin{tabular}{|c|c|c|c|}
\hline Gene & $\begin{array}{l}\text { Primer sequence } \\
\qquad\left(5^{\prime}-3^{\prime}\right)\end{array}$ & Annealing temperature $\left({ }^{\circ} \mathrm{C}\right)$ & Product size (bp) \\
\hline GAPDH & $\begin{array}{c}\text { Forward: GTGGTCTCCTCTGACTTCAACA } \\
\text { Reverse: CTCTTCCTCTTGTGCTCTTGCT }\end{array}$ & 62 & 210 \\
\hline OCT4 & $\begin{array}{l}\text { Forward: GACAACAATGAGAACCTTCAGGAGA } \\
\text { Reverse: TTCTGGCGCCGGTTACAGAACCA }\end{array}$ & 62 & 218 \\
\hline SOX2 & $\begin{array}{l}\text { Forward: AACCAAGACGCTCATGAAGAAG } \\
\text { Reverse: GCGAGTAGGACATGCTGTAGGT }\end{array}$ & 62 & 341 \\
\hline$c M Y C$ & $\begin{array}{l}\text { Forward: TCGGATTCTCTGCTCTCCTC } \\
\text { Reverse: CGCCTCTTGACATTCTCCTC }\end{array}$ & 62 & 413 \\
\hline KLF4 & $\begin{array}{l}\text { Forward: ATTCTCTCCAATTCGCTGACCC } \\
\text { Reverse: TTCAGCACGAACTTGCCCAT }\end{array}$ & 62 & 376 \\
\hline NANOG & $\begin{array}{l}\text { Forward: ATAGCAATGGTGTGACGCAG } \\
\text { Reverse: GATTGTTCCAGGATTGGGTG }\end{array}$ & 62 & 219 \\
\hline P53 & $\begin{array}{l}\text { Forward: TCGACATAGTGTGGTGGTGC } \\
\text { Reverse: TTGGACTTCAGGTGGCTGGA }\end{array}$ & 58 & 480 \\
\hline LIN28 & $\begin{array}{l}\text { Forward: GCTCCGTGTCCAACCAGCAG } \\
\text { Reverse: TTTCCTTTTGGCCGCCTCTC }\end{array}$ & 58 & 376 \\
\hline P16 & $\begin{array}{l}\text { Forward: CGAATAGTTACGGTCGGAGG } \\
\text { Reverse: GCATGGTTACTGCCTCTGGT }\end{array}$ & 62 & 309 \\
\hline$D L X 5$ & $\begin{array}{l}\text { Forward: ACCATCCGTCTCAGGAATCG } \\
\text { Reverse: ACCTTCTCTGTAATGCGGCC }\end{array}$ & 60 & 384 \\
\hline RUNX2 & $\begin{array}{l}\text { Forward: TTGCAGCCATAAGAGGGTAG } \\
\text { Reverse: GTCACTTTCTTGGAGCAGGA }\end{array}$ & 58 & 470 \\
\hline PPARG & $\begin{array}{l}\text { Forward: TCTCTCCGTAATGGAAGACC } \\
\text { Reverse: GCATTATGAGACATCCCCAC }\end{array}$ & 55 & 474 \\
\hline C/EBPA & $\begin{array}{l}\text { Forward: CCAAGAAGTCGGTGGACAAGAA } \\
\text { Reverse: TCATTGTCACTGGTCAGCTCCA }\end{array}$ & 62 & 145 \\
\hline$B M P 7$ & $\begin{array}{l}\text { Forward: CCAACGTCATCCTGAAGAAATAC } \\
\text { Reverse: GCTTGTAGGATCTTGTTCATTGG }\end{array}$ & 60 & 271 \\
\hline SOX9 & $\begin{array}{l}\text { Forward: GGTTGTTGGAGCTTTCCTCA } \\
\text { Reverse: TAGCCTCCСTCACTCCAAGA }\end{array}$ & 61 & 400 \\
\hline $\mathrm{P} 21$ & $\begin{array}{l}\text { Forward: GCGATGGAACTTCGACTTTG } \\
\text { Reverse: CGTTTTCGACCCTGAGAGAGTC }\end{array}$ & 60 & 285 \\
\hline OSTEOCALCIN & $\begin{array}{l}\text { Forward: CGCAGCCACCGAGACACCAT } \\
\text { Reverse: GGGCAAGGGCAAGGGGAAGA }\end{array}$ & 62 & 405 \\
\hline OSTEOPONTIN & $\begin{array}{l}\text { Forward: GAGACCCTTCCAAGTAAGTCCA } \\
\text { Reverse: GATGTCCTCGTCTGTAGCATCA }\end{array}$ & 62 & 354 \\
\hline COMP & $\begin{array}{l}\text { Forward: GAAGAACGACGACCAAAAGGAC } \\
\text { Reverse: GTCACAAGCATCTCCCACAAAG }\end{array}$ & 62 & 232 \\
\hline TYPE I COLLAGEN & $\begin{array}{l}\text { Forward: CACAGAGGTTTCAGTGGTTTGG } \\
\text { Reverse: GCACCAGTAGCACCATCATTTC }\end{array}$ & 62 & 191 \\
\hline$A P 2$ & $\begin{array}{l}\text { Forward: AAGAAGTAGGAGTGGGCTTTGC } \\
\text { Reverse: CCACCACCAGTTTATCATCCTC }\end{array}$ & 62 & 381 \\
\hline$L P L$ & $\begin{array}{l}\text { Forward: AGAGAGGACTTGGAGATGTGGA } \\
\text { Reverse: GGAAGACTTTGTAGGGCATCTG }\end{array}$ & 62 & 264 \\
\hline
\end{tabular}

preserve MSC functionality and would be beneficial for MSC culture in vitro.

The aim of this study was to examine the effects of glabridin on MSC in vitro culture. Treatment of MSCs with glabridin provided a favorable environment that improved stemness through upregulation of the OCT4 gene, which is involved in pluripotency. To investigate the effects of glabridin on the differentiation potential of MSCs, we examined genes for osteogenic factors (DLX5 and RUNX2), chondrogenic factors (BMP7 and SOX9), and adipogenic factors (PPARG and C/EBPA). We found that the treatment of MSCs with glabridin led to osteoblast differentiation with the upregulation of osteoblast markers such as OSTEOCALCIN and OSTEOPONTIN. To our knowledge, this is the first study to indicate that glabridin is a beneficial factor that induces the OCT4 gene and improves osteogenic 


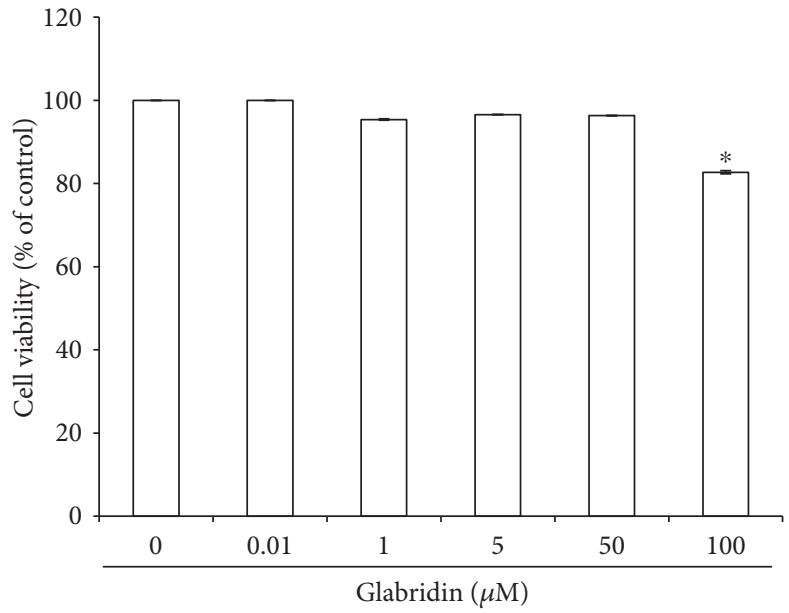

(a)

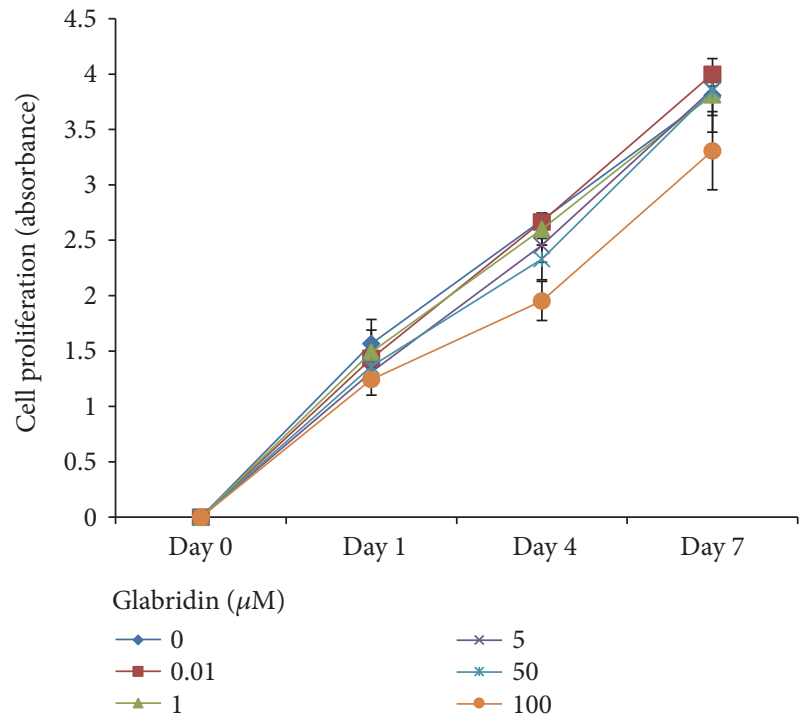

(b)

FIGURE 1: (a) Effect of glabridin on cell viability in bone marrow-derived mesenchymal stem cells (MSCs). Cell viability of MSCs treated with an increasing concentration of glabridin was determined by a CCK-8 assay. The data are expressed as the mean \pm SD of three independent experiments. ${ }^{*} P<0.05$ versus untreated control. (b) Growth rates of cultured MSCs. Cells were cultivated with an increasing concentration of glabridin for 7 days. Proliferation activity was measured using a CCK- 8 kit containing WST-8. The data are expressed as the mean \pm SD of three independent experiments.

differentiation of MSCs during culture in vitro. These findings will be useful for preparing highly functional MSCs for cell-based clinical applications.

\section{Materials and Methods}

2.1. Cell Culture. The bone marrow was collected from healthy donors after obtaining written informed consent. This study was approved by the Institutional Review Boards of Severance Hospital of Yonsei University Health System, Seoul, Korea. As previously described, mononuclear cells were isolated by Ficoll-Hypaque density gradient centrifugation (Pharmacia Biotech, Uppsala, Sweden), and the MSCs were cultured using the plastic adherence method [14]. The cells were maintained in DMEM Low Glucose supplemented with $10 \%$ fetal bovine serum (FBS) and $1 \%$ penicillin/streptomycin $(\mathrm{P} / \mathrm{S})$ at $37^{\circ} \mathrm{C}$ with $5 \% \mathrm{CO}_{2}$ (all from Invitrogen, Carlsbad, CA, USA). The medium was changed every 3 or 4 days. The cells were subcultured using $0.05 \%$ trypsin/EDTA (Invitrogen) when they reached approximately $80-90 \%$ confluence. Glabridin $(0.01-100 \mu \mathrm{M})$ was purchased from Sigma-Aldrich (St. Louis, MO, USA).

2.2. Cell Viability Test. The cells were seeded into 12-well plates (Corning Inc., Corning, NY, USA) at a density of $4 \times 10^{4}$ cells/well for assessment of cell viability. The next day, cells were treated with glabridin $(0.01-100 \mu \mathrm{M})$, directly added to the medium, for $24 \mathrm{~h}$. The viability of the cells was analyzed using a CCK-8 kit (Dojindo Co., Kumamoto, Japan), which measures cell metabolic activity, according to the manufacturer's instructions [15]. Briefly, $50 \mu \mathrm{M}$ of the CCK-8 solution was added to each well of the culture plate at the end of the culture period. After $4 \mathrm{~h}$ of incubation, absorbance was measured at $450 \mathrm{~nm}$. Cells incubated without glabridin were used as a control.

2.3. Cell Proliferation Assay. Cells were plated at a density of $2 \times 10^{4} /$ well in 12-well plates (Corning) for analysis of cell growth. When the cells were replated, glabridin (0.01-100 $\mu \mathrm{M})$ was added to each well of the culture plate. A proliferation assay was performed using a CCK- 8 kit. CCK-8 contains WST-8 [2-(2-methoxy-4-nitrophenyl)3-(4-nitrophenyl)-5-(2,4-dissulfophenyl)-2H-tetrazolium, monosodium salt], which produces a water-soluble formazan dye upon reduction in the presence of an electron carrier. CCK-8, being nonradioactive, allows sensitive colorimetric assays for the determination of the number of viable cells during cell proliferation. Cultures were maintained for 7 days and then analyzed for cell growth on days 1, 4, and 7 according to the manufacturer's instructions. Cells incubated with the culture medium alone were used as a control. The absorbance of the cells was normalized to their respective day 0 absorbance.

2.4. Reverse Transcription PCR (RT-PCR). Total RNA was isolated using TRIzol reagent (Invitrogen). Standard reverse transcription was performed using transcriptase II (Invitrogen). RT-PCR was performed using PCR primers (Bioneer, Daejeon, Korea) under the conditions listed in Table 1. Glyceraldehyde 3-phosphate dehydrogenase (GAPDH) level was used as an internal control. The signal intensity of the product was normalized to its respective GAPDH signal intensity. 


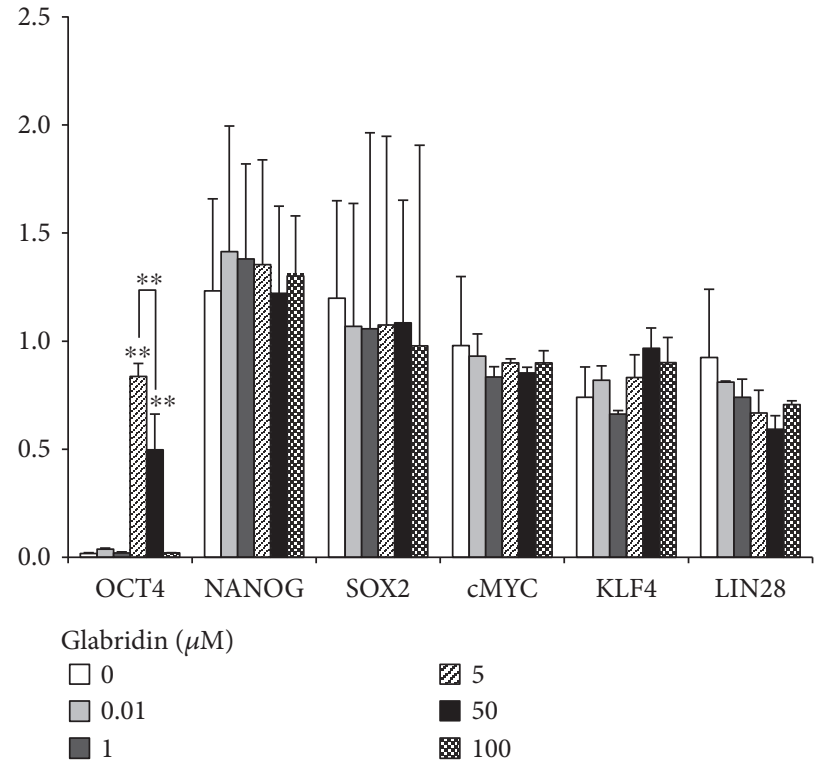

(a)

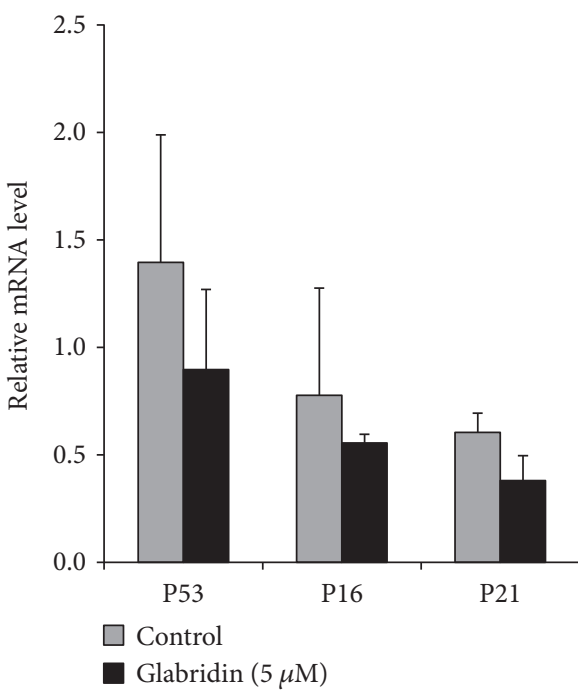

(c)

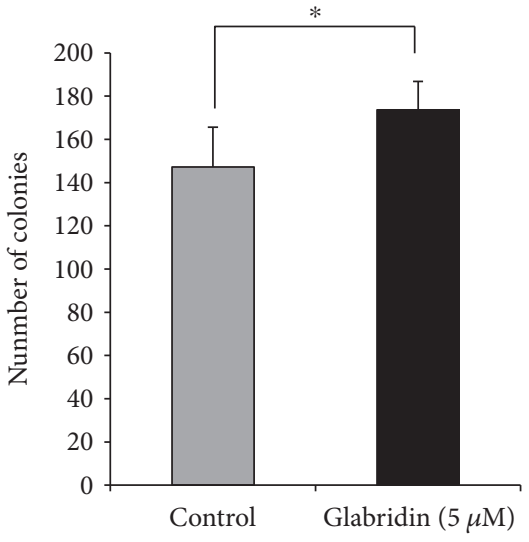

(b)

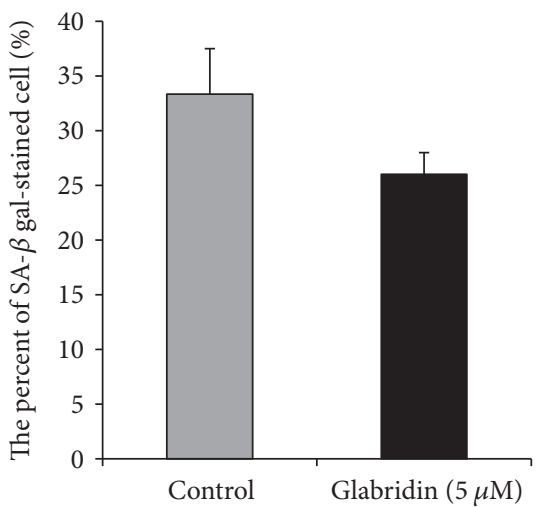

(d)

FIGURE 2: Effect of glabridin on stemness and senescence in MSCs. (a) Stemness marker expression in MSCs treated with an increasing concentration of glabridin. (b) Stemness was evaluated by a CFU-F assay. The number of colonies ( $>50$ cells) was counted. (c) P53, P16, and P21 mRNA expression levels were analyzed using reverse transcription PCR (RT-PCR). (d) Senescence-associated (SA) $\beta$-gal assay. The number of $\beta$-gal-positive cells was counted. The data are expressed as the mean \pm SD of three experiments. ${ }^{*} P<0.05$ and ${ }^{* *} P<0.01$ versus untreated control.

2.5. Colony Forming-Unit-Fibroblast (CFU-F) Assay. For assessment of self-renewal properties, a CFU-F assay was performed. Briefly, $1 \times 10^{3}$ cells were plated in $100 \mathrm{~mm}$ dishes (Corning), and the cells were cultured for 14 days. Following incubation for 14 days, the cells were washed with phosphate-buffered saline (PBS; Invitrogen). Then, the cells were stained with $0.5 \%$ crystal violet (SigmaAldrich) for $5 \mathrm{~min}$ at room temperature, and stained colonies were counted.

2.6. $\beta$-Galactosidase Staining. Senescent cells show an increase in cell size and the senescence-associated expression of $\beta$-galactosidase activity. A senescence detection kit (BioVision Inc., CA, USA) was used to histochemically detect $\beta$-galactosidase activity in cultured cells, according to the manufacturer's instructions. Briefly, cultured cells were washed with PBS and fixed with 4\% paraformaldehyde at room temperature. After washing with PBS, cells were incubated with $\beta$-galactosidase staining solution for $24 \mathrm{~h}$ at $37^{\circ} \mathrm{C}$. The number of $\beta$-galactosidase-stained cells was counted under a light microscope (Olympus-IX71; Olympus, Tokyo, Japan).

2.7. Differentiation Assay. To induce MSC differentiation into osteoblasts, chondrocytes, and adipocytes, cells were cultured in osteogenic induction medium, chondrogenic 


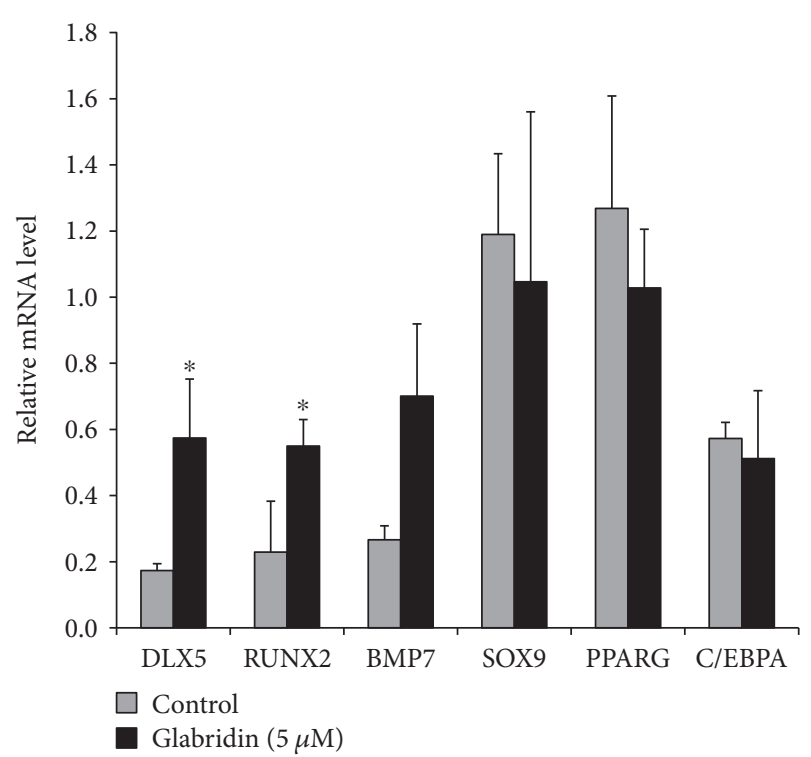

Figure 3: Gene expression in MSCs following glabridin treatment. RT-PCR analysis of osteogenic, chondrogenic, and adipogenic markers was performed in control and glabridin-treated MSCs. Relative mRNA expression levels of trilineage-associated genes in the control and glabridin-treated MSCs. Expression levels relative to GAPDH are shown. The data are expressed as the mean $\pm \mathrm{SD}$ of three experiments. ${ }^{*} P<0.05$ versus untreated control.

induction medium, or adipogenic induction medium for 3 weeks (Cambrex, Lonza, MD, USA), respectively. The medium was changed every 3 or 4 days, and the cells for chondrogenic differentiation were treated with $10 \mathrm{ng} / \mathrm{ml}$ transforming growth factor (TGF)- $\beta 3$ (Cambrex) whenever the medium was replaced. The induced cells were stained with von Kossa to confirm osteogenesis, safranin O to confirm chondrogenesis, and oil red $\mathrm{O}$ to confirm adipogenesis. Images of the stained cells were taken using a phase microscope (Olympus-IX-71). To measure the calcium content in osteogenesis, the Calcium LiquiColor kit (Stanbio Laboratory, Boerne, USA) was used according to a previously described method [16]. Briefly, the cells were washed with PBS and treated with $0.5 \mathrm{~N} \mathrm{HCl}$. After shaking for $3 \mathrm{~h}$ by using an orbital shaker, the supernatant was transferred to a new tube for analysis. Ortho-cresolphthalein complexone (OCPC) was added to the sample, and absorbance was determined at $550 \mathrm{~nm}$. For quantitative analysis of adipogenesis, absorbance was measured at $500 \mathrm{~nm}$ after destaining with isopropanol for $30 \mathrm{~min}$ according to a previously reported method [16]. To quantitatively evaluate chondrogenesis, the absorbance of sulfated glycosaminoglycan was measured at $656 \mathrm{~nm}$ by using the Blyscan assay kit (Biocolor Ltd., County Antrim, UK). Briefly, the supernatant was transferred to a new tube and each sample was mixed with 1,9-dimethylmethylene blue (DMMB) dye, which is used to measure sulfated glycosaminoglycan (sGAG) content, according to the manufacturer's instructions and a previous report [17].

2.8. Statistical Analysis. Quantitative data are expressed as the means \pm standard deviation (SD). Statistical comparisons were performed by a Student's $t$-test and one-way analysis of variance (ANOVA) with post hoc Bonferroni corrections. The differences were considered statistically significant at $P<0.05$. Statistical analyses were performed using SPSS software (SPSS Inc., Chicago, IL, USA).

\section{Results}

3.1. Effect of Glabridin on the Viability and Proliferation of MSCs. To investigate the effect of glabridin on MSC survival, cells were cultured with increasing concentrations (0.01$100 \mu \mathrm{M}$ ) of glabridin for $24 \mathrm{~h}$ and then cell viability was measured using a CCK- 8 assay. Glabridin at concentrations of $0.01-50 \mu \mathrm{M}$ had no effect on cell survival, whereas incubation with $100 \mu \mathrm{M}$ glabridin decreased the cell viability of MSCs (Figure 1(a)). These results show that $100 \mu \mathrm{M}$ glabridin itself was cytotoxic to MSCs.

To evaluate the effect of glabridin on MSC proliferation, MSCs were cultured for 7 days to determine whether glabridin stimulates MSC growth, and then cell proliferation was determined using the CCK-8 assay. A decrease in cell growth was detected in MSCs cultured with $100 \mu \mathrm{M}$ glabridin, while the cells cultured with $0.01-50 \mu \mathrm{M}$ glabridin exhibited proliferation properties similar to those of the control cells (Figure 1(b)). These results show that glabridin did not facilitate the growth rates of the MSCs.

3.2. Effect of Glabridin on MSC Stemness. In order to identify the self-renewal capacity of MSCs, we analyzed the expression levels of stemness markers in the MSCs cultured with 0.01-100 $\mu \mathrm{M}$ glabridin. NANOG, SOX2, $c M Y C, K L F 4$, and LIN28 were similarly expressed with all concentrations of glabridin (Figure 2(a)). Interestingly, the OCT4 gene-involved in the self-renewal of undifferentiated stem cells-was markedly detected in MSCs cultured with $5 \mu \mathrm{M}$ glabridin (Figure 2(a)). Therefore, we chose this concentration of glabridin $(5 \mu \mathrm{M})$ for all subsequent cell experiments. The CFU-F assay was used to investigate whether OCT4 expression with glabridin promotes the self-renewal capacity of MSCs. Glabridin significantly enhanced the self-renewal capacity of the treated cells compared with that of the control cells (Figure 2(b)). In addition, to confirm whether glabridin affected the cell cycle, the mRNA expression levels of P53, $P 16^{I N K 4 a}$, and $P 21^{C i p 1}$ in MSCs treated with glabridin were measured. The expression levels of P53, P16 ${ }^{I N K 4 a}$, and $P 21^{C i p 1}$ of MSCs treated with glabridin were decreased compared to those in control cells (Figure 2(c)). We next examined whether glabridin prevented MSC senescence. MSCs treated with glabridin showed a decrease in the percentage of $\beta$-galactosidase-stained cells compared to control cells although there was no significance (Figure 2(d)). Together, these results suggest that glabridin augments the selfrenewal capacity with upregulation of the OCT4 gene.

3.3. Changes of Differentiation Potential in MSCs by Glabridin. We subsequently examined the osteogenesis-, chondrogenesis-, and adipogenesis-related gene expression levels in the MSCs after glabridin treatment. DLX5 and RUNX2 genes, which are involved in osteogenesis, were 

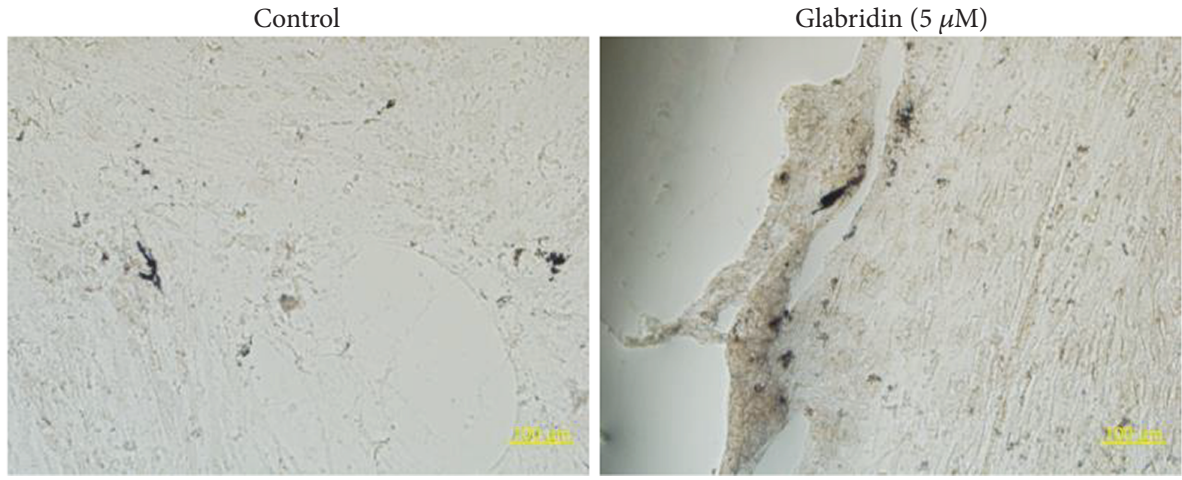

(a)

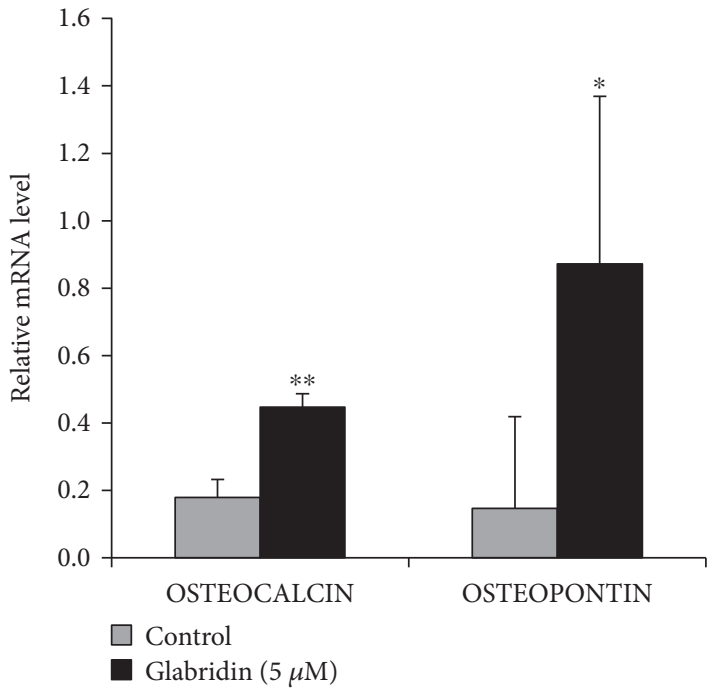

(b)

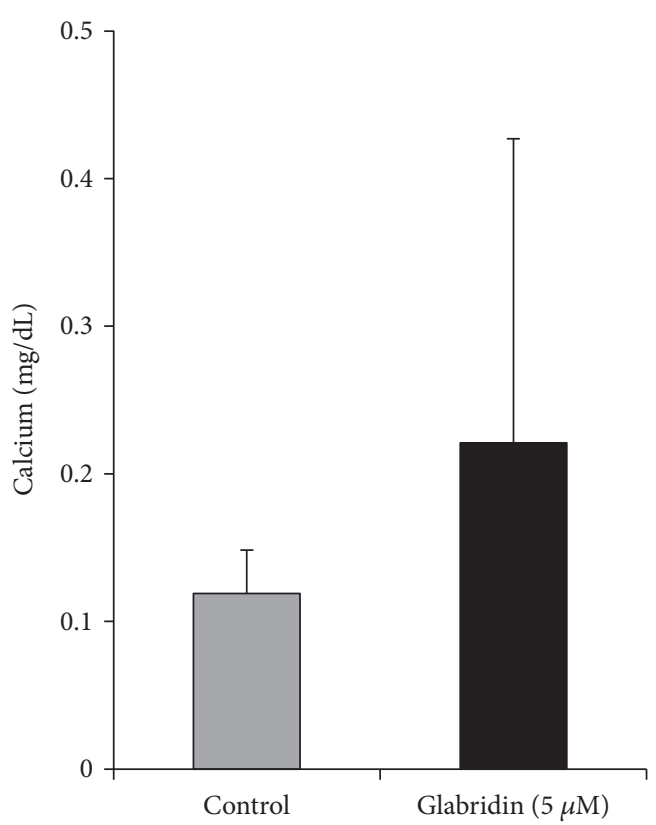

(c)

FIGURE 4: Effect of glabridin on osteogenesis in MSCs. (a) Osteogenic differentiation was evaluated by von Kossa staining (magnification: 200x). (b) Osteogenic potential was analyzed by OSTEOCALCIN and OSTEOPONTIN gene expression using RT-PCR. (c) Differentiation of MSCs into osteoblasts was determined by calcium quantification. The data are expressed as the mean \pm SD of three experiments. ${ }^{*} P<0.05$ and ${ }^{* *} P<0.01$ versus untreated control.

markedly upregulated by glabridin (Figure 3 ). With respect to chondrogenesis, the $B M P 7$ gene was upregulated, whereas the expression of SOX9 gene was similar to MSCs treated with glabridin (Figure 3). The adipogenesis-related $C / E B P A$ gene was similarly expressed in glabridin-treated MSCs compared to that in control cells, while the expression level of the PPARG gene decreased with glabridin treatment (Figure 3). These results indicate that glabridin strongly affects the osteogenic potential of MSCs.

3.4. Glabridin Enhances Osteogenesis of MSCs. To evaluate the differentiation capacity of MSCs, cells were induced by glabridin to form osteoblasts, chondrocytes, and adipocytes. MSCs treated with glabridin showed higher amounts of von Kossa staining, which detects calcium-containing mineralized nodules, compared to control cells (Figure 4(a)), and the expression of OSTEOCALCIN and OSTEOPONTIN genes of osteogenic differentiation markers was upregulated in MSCs treated with glabridin (Figure 4(b)). Moreover, we confirmed that MSCs cultured with glabridin had a higher degree of calcium accumulation compared to control cells although there were no significant differences (Figure 4(c)). Chondrogenesis was assessed by safranin $\mathrm{O}$ staining. After chondrogenic induction, MSCs cultured with glabridin exhibited a slightly higher chondrogenic differentiation capacity (Figure 5(a)). However, the COMP and the TYPE I COLLAGEN genes of the chondrogenic differentiation markers were similarly expressed despite chondrogenic induction in both conditions (Figure 5(b)). Sulfated glycosaminoglycan content was slightly increased in MSCs grown with glabridin, irrespective of the PCR data (Figure 5(c)). There were no significant differences. In adipogenesis, glabridin slightly 

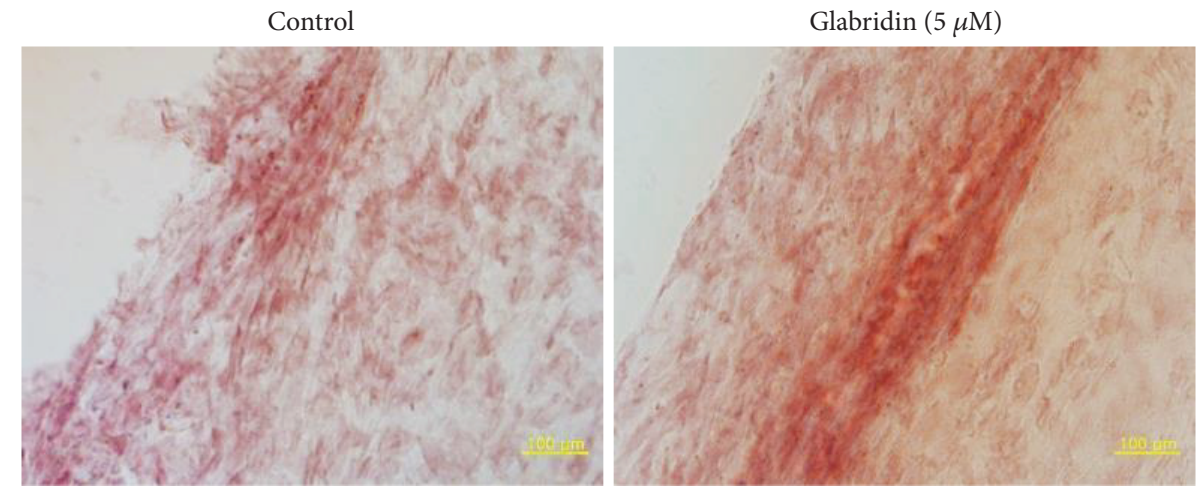

(a)
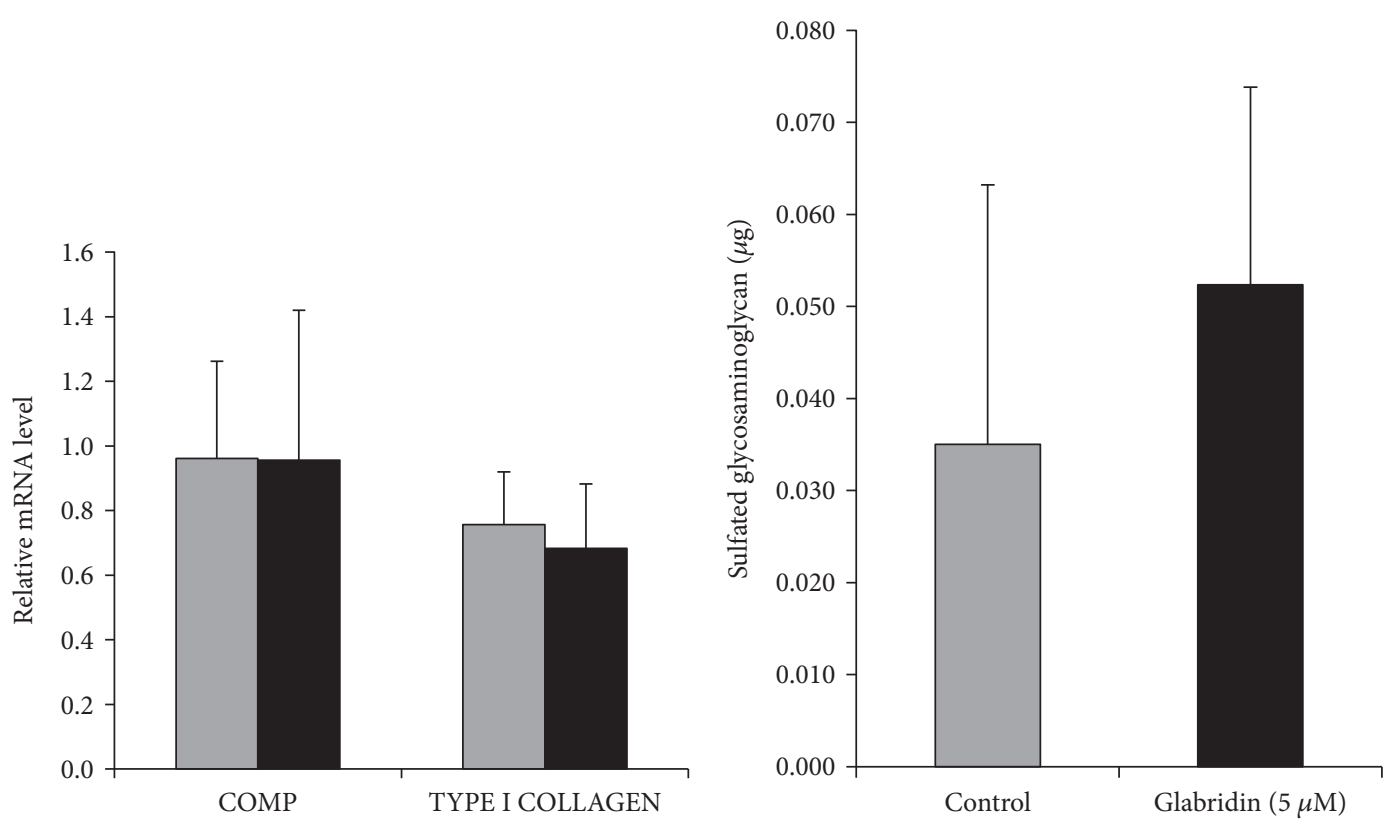

$\square$ Control

- Glabridin $(5 \mu \mathrm{M})$

(b)

(c)

Figure 5: Effect of glabridin on chondrogenesis in MSCs. (a) Chondrogenic differentiation was evaluated by safranin O staining (magnification: 200x). (b) Chondrogenic potential was analyzed by levels of COMP and TYPE I COLLAGEN gene expression using RT-PCR. (c) Differentiation of MSCs into chondrocytes was determined by glycosaminoglycan quantification. The data are expressed as the mean $\pm S D$ of three experiments.

suppressed adipogenic differentiation as shown in Figure 6(a). In addition, the AP2 and LPL genes of adipogenesis-related markers were slightly decreased in glabridin-treated cells without a significant difference (Figure 6(b)). We also confirmed that the absorbance value of lipid droplets was reduced in MSCs induced with glabridin (Figure 6(c)). Together, these results imply that glabridin prominently enhanced the osteogenic differentiation capacity of MSCs by upregulating the OSTEOCALCIN and OSTEOPONTIN genes.

\section{Discussion}

Of the adult stem cells, MSCs have been widely used for clinical applications because of their plastic and antiinflammatory effects $[18,19]$. Although MSCs represent a new approach to treat intractable diseases, clinical trials using these cells have been impeded by low quantities of cells and difficulty of cell culture, described as cellular or replicative senescence. MSCs can easily enter a state of growth arrest, known as senescence, despite high self-renewal capacity by internal and/or external stimuli [5]. Therefore, the culture and maintenance of MSCs without the loss of stemness are very critical for their extensive clinical use.

In general, cells are affected by multiple biochemical and biophysical factors such as the extracellular matrix (ECM) and soluble factors $[20,21]$. Previously, we prevented senescence and augmented MSC growth using poly-L-lysine (PLL) of ECM proteins [22]. PLL definitely improved the proliferation capacity and functionality of MSCs. However, using PLL as a coating substrate is time-consuming because culture vessels should be incubated and dried for a long time 


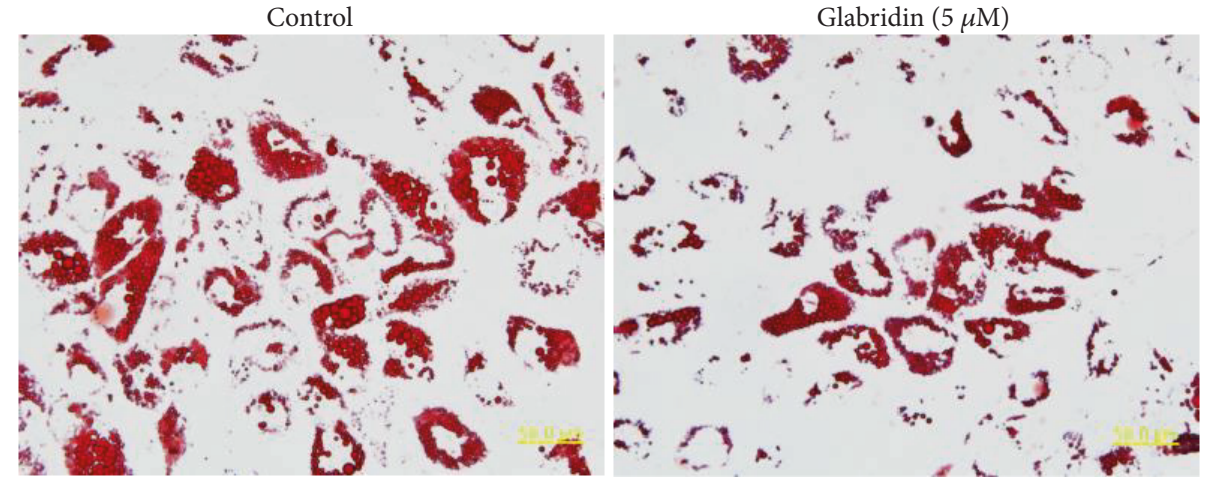

(a)

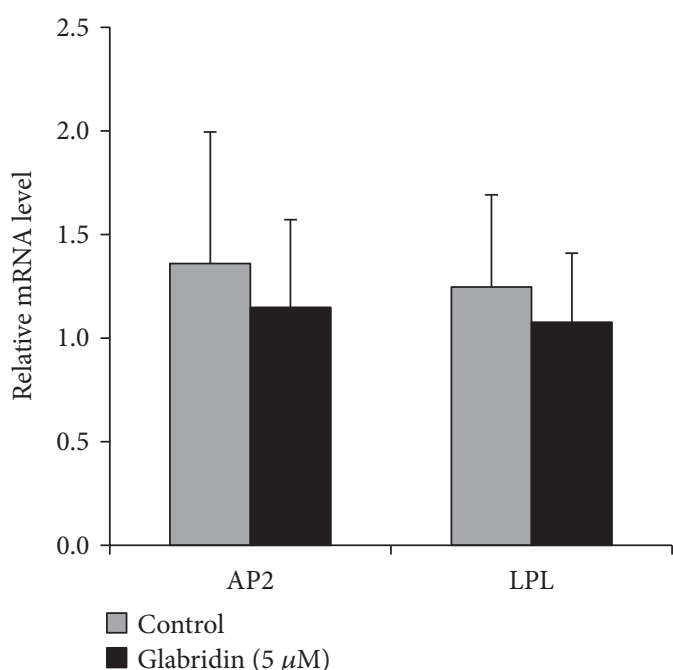

(b)

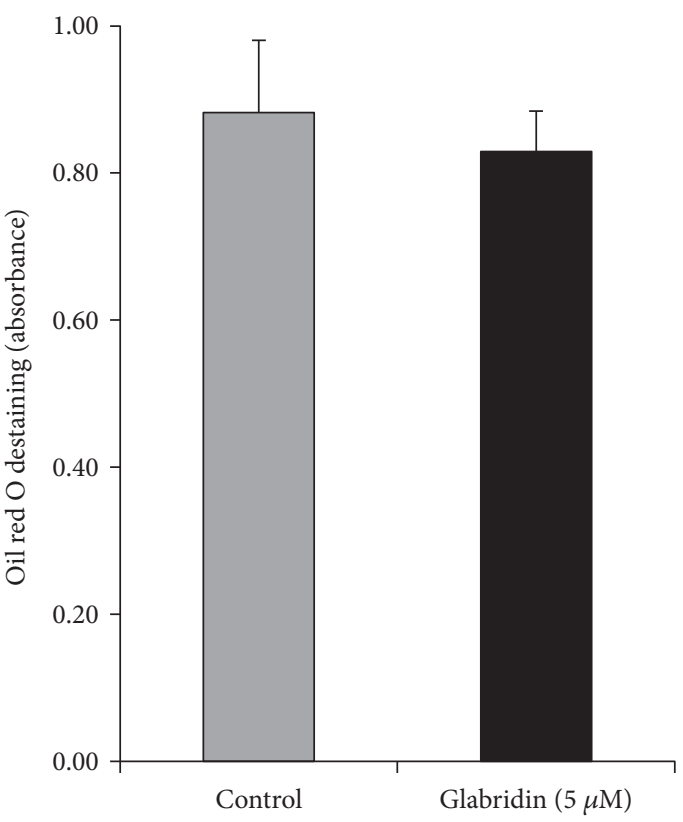

(c)

FIGURE 6: Effect of glabridin on adipogenesis in MSCs. (a) Adipogenic differentiation was evaluated by oil red O staining (magnification: 400x). (b) Adipogenic capacity was analyzed by AP2 and LPL mRNA expression using RT-PCR. (c) Absorbance was determined after oil red $\mathrm{O}$ destaining for quantitative analysis. The data are expressed as the mean \pm SD of three experiments.

after PLL coating. Recently, Kim et al. reported that glabridin, one of the major active flavonoids in licorice, attenuates oxidative damage and improves osteogenic differentiation function [13]. It was also reported that glabridin inhibits the cancer stem cell-like properties in hepatocellular carcinoma cells [23]. Cellular senescence is very closely related to oxidative stress [24]. In the present study, we applied glabridin, which has an antioxidant activity, to a cell culture condition. We found that glabridin did not significantly affect the viability of bone marrow-derived MSCs. In addition, glabridin did not activate cell proliferation in in vitro culture. We analyzed the expression levels of OCT4, NANOG, SOX2, cMYC, KLF4, and LIN28 genes known as stemness markers of stem cells to investigate molecular patterns of selfrenewal capacity. NANOG, SOX2, cMYC, KLF4, and LIN28, which are involved in pluripotency and self-renewal of stem cells, were similarly expressed in all conditions. OCT4, an essential transcription factor in the maintenance of pluripotency, is expressed in embryonic stem cells [25]. In addition, OCT4 is a very important gene for the generation of induced pluripotent stem cells [26]. It is known that OCT4, as a specific marker of embryonic stem cells, is also expressed in MSCs [27]. However, it is hard to detect the expression of OCT4 in MSCs because it readily disappears during culture in vitro [28]. Recently, Piccinato et al. showed that a high OCT4 gene expression might be a potential hallmark and predictor of greater in vitro lifespan and growth potential of MSCs [29]. These results indicate that the expression level of the OCT4 gene may be a specific factor that affects MSC senescence.

In this study, the OCT4 gene was strongly induced in the presence of $5 \mu \mathrm{M}$ glabridin. However, there was no dosedependent OCT4 expression by glabridin. Rather, MSCs treated with $100 \mu \mathrm{M}$ glabridin did not express OCT4. The 


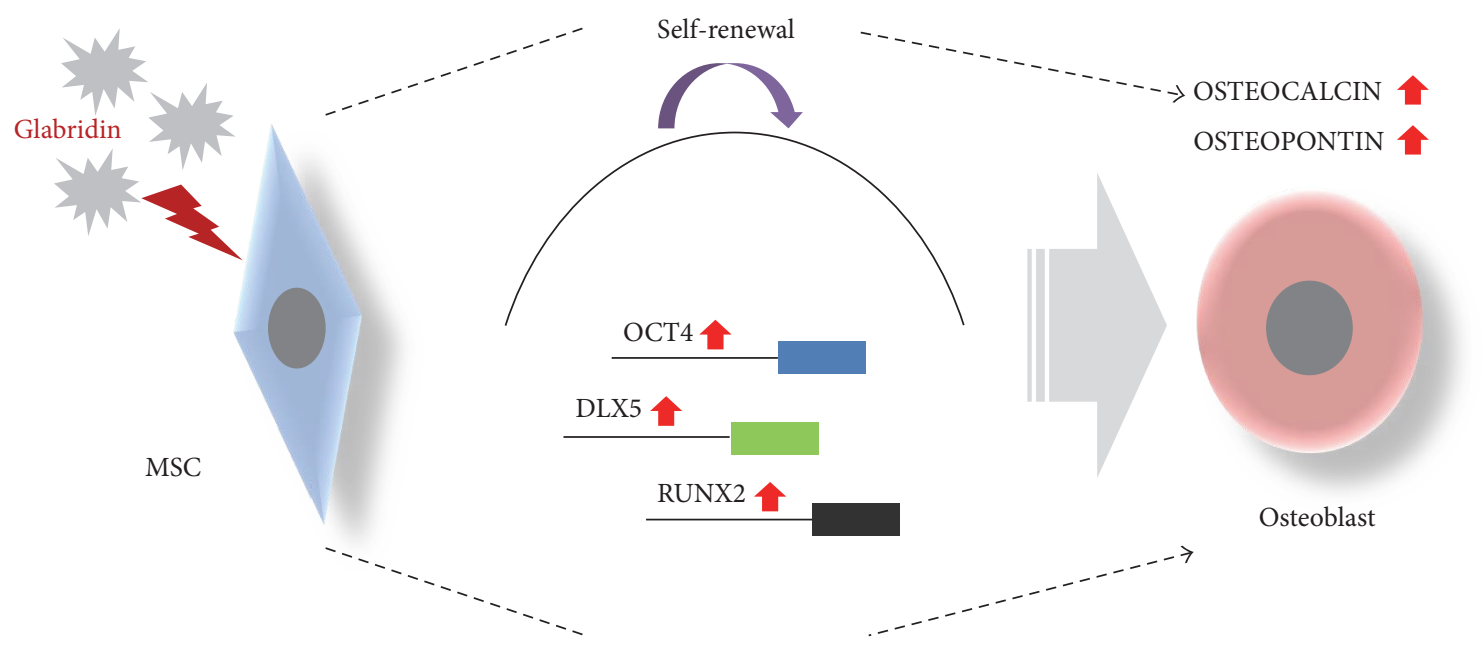

FIGURE 7: Schematic summary of the effect of glabridin on osteogenesis of MSCs. Our results suggest that glabridin upregulates the expression level of the OCT4 gene associated with stemness and that of DLX5 and RUNX2 genes related to osteogenesis. Glabridin enhances OCT4induced osteogenesis of MSCs by activating OSTEOCALCIN and OSTEOPONTIN genes for osteogenic differentiation.

expression of OCT4 might be suppressed by a decrease in viability as shown in Figure 1. When MSCs were treated with glabridin, a significant increase in their self-renewal capacity was observed, implying that glabridin enhances CFU-F capacity by inducing the OCT4 gene. Several studies have reported that cellular senescence is regulated by the $P 53$, $P 16^{I N K 4 a}$, and $P 21^{C i p 1}$ pathways via the accumulation of reactive oxygen species [30-32]. We have confirmed that the P53, $P 16^{I N K 4 a}$, and $P 21^{C i p 1}$ mRNA expression levels were inhibited by glabridin although there was no significance. These results coincide with previous results, demonstrating that cellular senescence of MSCs could be suppressed via inhibition of $P 53$ and $P 21^{C i p 1}$ [33]. In addition, glabridin delayed MSC senescence as observed by $\beta$-galactosidase staining, showing that glabridin positively affects cell senescence in MSCs cultured in vitro. Together, these results strongly support that glabridin has antisenescence effects.

Recently, it was reported that MSC treatment with glabridin resulted in a significant elevation of alkaline phosphatase (ALP) activity, collagen content, and expression of osteoblast differentiation genes [13]. To investigate the effects of glabridin on MSC differentiation potential, trilineage (osteogenesis, chondrogenesis, adipogenesis)-related key transcription factors were analyzed by RT-PCR after treatment with glabridin as compared with control cells. When MSCs were treated with glabridin, significant increases in the gene expression of DLX5 and RUNX2 for osteogenesis were observed. In the differentiation assay, our results demonstrated that glabridin could significantly improve osteogenic differentiation capacity with significant upregulation of OSTEOCALCIN and OSTEOPONTIN genes of osteogenesis markers. Regarding chondrogenesis, sulfated glycosaminoglycan contents were also elevated by glabridin, but glabridin had no effects on the chondrogenic differentiation marker genes. Moreover, glabridin slightly attenuated adipogenic differentiation capacity with changes in the expression levels of genes associated with adipogenesis. These results correspond to a previous result, demonstrating that overexpression of DLX5, a key factor for osteogenesis, inhibited the expression of adipogenic marker genes [34]. In the present results, enhancement of osteogenesis inhibited adipogenesis of human MSCs, due to the reverse relationship between osteogenic and adipogenic differentiation.

In summary, we have shown that glabridin improved osteogenic differentiation capacity of MSCs by inducing the expression of the OCT4 gene of the pluripotency factors and augmenting DLX5 and RUNX2 gene expression for osteogenesis as shown in Figure 7. We thus conclude that glabridin could be used in the MSC culture system in vitro. Furthermore, MSC culture using glabridin will contribute greatly to regenerative medicine and cell-based therapies including bone diseases.

\section{Conflicts of Interest}

The authors declare no conflicts of interest. The authors have no commercial, proprietary, or financial interest in the products or companies described in this article.

\section{References}

[1] P. Bianco, M. Riminucci, S. Gronthos, and P. G. Robey, "Bone marrow stromal stem cells: nature, biology, and potential applications," Stem Cells, vol. 19, no. 3, pp. 180-192, 2001.

[2] G. Chamberlain, J. Fox, B. Ashton, and J. Middleton, "Concise review: mesenchymal stem cells: their phenotype, differentiation capacity, immunological features, and potential for homing," Stem Cells, vol. 25, no. 11, pp. 2739-2749, 2007.

[3] A. I. Caplan and J. E. Dennis, "Mesenchymal stem cells as trophic mediators," Journal of Cellular Biochemistry, vol. 98, no. 5, pp. 1076-1084, 2006.

[4] P. H. Lee, J. E. Lee, H. S. Kim et al., "A randomized trial of mesenchymal stem cells in multiple system atrophy," Annals of Neurology, vol. 72, no. 1, pp. 32-40, 2012.

[5] K. Itahana, J. Campisi, and G. P. Dimri, "Mechanisms of cellular senescence in human and mouse cells," Biogerontology, vol. 5, no. 1, pp. 1-10, 2004. 
[6] J. S. Lee, M. O. Lee, B. H. Moon, S. H. Shim, A. J. Fornace Jr., and H. J. Cha, "Senescent growth arrest in mesenchymal stem cells is bypassed by Wip1-mediated downregulation of intrinsic stress signaling pathways," Stem Cells, vol. 27, no. 8, pp. 1963-1975, 2009.

[7] S. Loft, P. Hogh Danielsen, L. Mikkelsen, L. Risom, L. Forchhammer, and P. Moller, "Biomarkers of oxidative damage to DNA and repair," Biochemical Society Transactions, vol. 36, no. 5, pp. 1071-1076, 2008.

[8] M. M. Bonab, K. Alimoghaddam, F. Talebian, S. H. Ghaffari, A. Ghavamzadeh, and B. Nikbin, "Aging of mesenchymal stem cell in vitro,” BMC Cell Biology, vol. 7, p. 14, 2006.

[9] Y. W. Chin, H. A. Jung, Y. Liu et al., "Anti-oxidant constituents of the roots and stolons of licorice (Glycyrrhiza glabra)," Journal of Agricultural and Food Chemistry, vol. 55, no. 12, pp. 4691-4697, 2007.

[10] E. M. Choi, "Glabridin protects osteoblastic MC3T3-E1 cells against antimycin A induced cytotoxicity," Chemico-Biological Interactions, vol. 193, no. 1, pp. 71-78, 2011.

[11] H. Haraguchi, N. Yoshida, H. Ishikawa, Y. Tamura, K. Mizutani, and T. Kinoshita, "Protection of mitochondrial functions against oxidative stresses by isoflavans from Glycyrrhiza glabra," Journal of Pharmacy and Pharmacology, vol. 52, no. 2, pp. 219-223, 2000.

[12] F. Jiang, Y. Li, J. Mu et al., "Glabridin inhibits cancer stem cell-like properties of human breast cancer cells: an epigenetic regulation of miR-148a/SMAd2 signaling," Molecular Carcinogenesis, vol. 55, no. 5, pp. 929-940, 2016.

[13] H. S. Kim, K. S. Suh, A. Ko et al., "The flavonoid glabridin attenuates 2-deoxy-D-ribose-induced oxidative damage and cellular dysfunction in MC3T3-E1 osteoblastic cells," International Journal of Molecular Medicine, vol. 31, no. 1, pp. 243251, 2013.

[14] H. S. Sohn, J. S. Heo, H. S. Kim, Y. Choi, and H. O. Kim, "Duration of in vitro storage affects the key stem cell features of human bone marrow-derived mesenchymal stromal cells for clinical transplantation," Cytotherapy, vol. 15, no. 4, pp. 460-466, 2013.

[15] K. S. Suh, S. Chon, S. Oh et al., "Prooxidative effects of green tea polyphenol (-)-epigallocatechin-3-gallate on the HITT15 pancreatic beta cell line," Cell Biology and Toxicology, vol. 26, no. 3, pp. 189-199, 2010.

[16] Y. H. Kim, D. S. Yoon, H. O. Kim, and J. W. Lee, "Characterization of different subpopulations from bone marrow-derived mesenchymal stromal cells by alkaline phosphatase expression," Stem Cells and Development, vol. 21, no. 16, pp. 29582968, 2012.

[17] L. X. Tay, C. K. Lim, A. Mansor, and T. Kamarul, "Differential protein expression between chondrogenic differentiated MSCs, undifferentiated MSCs and adult chondrocytes derived from Oryctolagus cuniculus in vitro," International Journal of Medical Sciences, vol. 11, no. 1, pp. 24-33, 2014.

[18] G. H. Cui, Y. Y. Wang, C. J. Li, C. H. Shi, and W. S. Wang, "Efficacy of mesenchymal stem cells in treating patients with osteoarthritis of the knee: a meta-analysis," Experimental and Therapeutic Medicine, vol. 12, no. 5, pp. 3390-3400, 2016.

[19] E. Sykova, P. Rychmach, I. Drahoradova et al., “Transplantation of mesenchymal stromal cells in patients with amyotrophic lateral sclerosis: results of phase I/IIa clinical trial," Cell Transplantation, vol. 26, no. 4, pp. 647-658, 2016.
[20] Y. Xu, X. Zhu, H. S. Hahm et al., "Revealing a core signaling regulatory mechanism for pluripotent stem cell survival and self-renewal by small molecules," Proceedings of the National Academy of Sciences of the Unites States of America, vol. 107, no. 18, pp. 8129-8134, 2010.

[21] J. You, D. S. Shin, D. Patel, Y. Gao, and A. Revzin, "Multilayered heparin hydrogel microwells for cultivation of primary hepatocytes," Advanced Healthcare Materials, vol. 3, no. 1, pp. 126-132, 2014.

[22] J. S. Heo, H. O. Kim, S. Y. Song, D. H. Lew, Y. Choi, and S. Kim, "Poly-L-lysine prevents senescence and augments growth in culturing mesenchymal stem cells ex vivo," BioMed Research International, vol. 2016, Article ID 8196078, 13 pages, 2016.

[23] F. Jiang, J. Mu, X. Wang et al., "The repressive effect of miR148 a on TGF beta-SMADs signal pathway is involved in the glabridin-induced inhibition of the cancer stem cells-like properties in hepatocellular carcinoma cells," PLoS One, vol. 9, no. 5, article e96698, 2014.

[24] V. Turinetto, E. Vitale, and C. Giachino, "Senescence in human mesenchymal stem cells: functional changes and implications in stem cell-based therapy," International Journal of Molecular Sciences, vol. 17, no. 7, 2016.

[25] D. J. Rodda, J. L. Chew, L. H. Lim et al., "Transcriptional regulation of Nanog by OCT4 and SOX2," Journal of Biological Chemistry, vol. 280, no. 26, pp. 24731-24737, 2005.

[26] K. Takahashi, K. Tanabe, M. Ohnuki et al., "Induction of pluripotent stem cells from adult human fibroblasts by defined factors," Cell, vol. 131, no. 5, pp. 861-872, 2007.

[27] J. M. Lee, J. Jung, H. J. Lee et al., “Comparison of immunomodulatory effects of placenta mesenchymal stem cells with bone marrow and adipose mesenchymal stem cells," International Immunopharmacology, vol. 13, no. 2, pp. 219-224, 2012.

[28] T. M. Liu, Y. N. Wu, X. M. Guo, J. H. Hui, E. H. Lee, and B. Lim, "Effects of ectopic Nanog and Oct4 overexpression on mesenchymal stem cells," Stem Cells and Development, vol. 18, no. 7, pp. 1013-1021, 2009.

[29] C. A. Piccinato, A. L. Sertie, N. Torres, M. Ferretti, and E. Antonioli, "High OCT4 and low p $16^{\mathrm{INK} 4 \mathrm{~A}}$ expressions determine in vitro lifespan of mesenchymal stem cells," Stem Cells International, vol. 2015, Article ID 369828, 11 pages, 2015.

[30] U. Herbig, W. A. Jobling, B. P. Chen, D. J. Chen, and J. M. Sedivy, "Telomere shortening triggers senescence of human cells through a pathway involving ATM, p53, and p21 ${ }^{\mathrm{CIP1}}$, but not p16 ${ }^{\mathrm{INK} 4 \mathrm{a}}$," Molecular Cell, vol. 14, no. 4, pp. 501-513, 2004.

[31] K. Ksiazek, "A comprehensive review on mesenchymal stem cell growth and senescence," Rejuvenation Research, vol. 12, no. 2, pp. 105-116, 2009.

[32] Z. Tothova, R. Kollipara, B. J. Huntly et al., "FoxOs are critical mediators of hematopoietic stem cell resistance to physiologic oxidative stress," Cell, vol. 128, no. 2, pp. 325-339, 2007.

[33] T. Ito, R. Sawada, Y. Fujiwara, Y. Seyama, and T. Tsuchiya, "FGF-2 suppresses cellular senescence of human mesenchymal stem cells by down-regulation of TGF- $\beta 2$," Biochemical and Biophysical Research Communications, vol. 359, no. 1, pp. 108-114, 2007.

[34] H. L. Lee, K. M. Woo, H. M. Ryoo, and J. H. Baek, "Distal-less homeobox 5 inhibits adipogenic differentiation through the down-regulation of peroxisome proliferator-activated receptor $\gamma$ expression," Journal of Cellular Physiology, vol. 228, no. 1, pp. 87-98, 2013. 

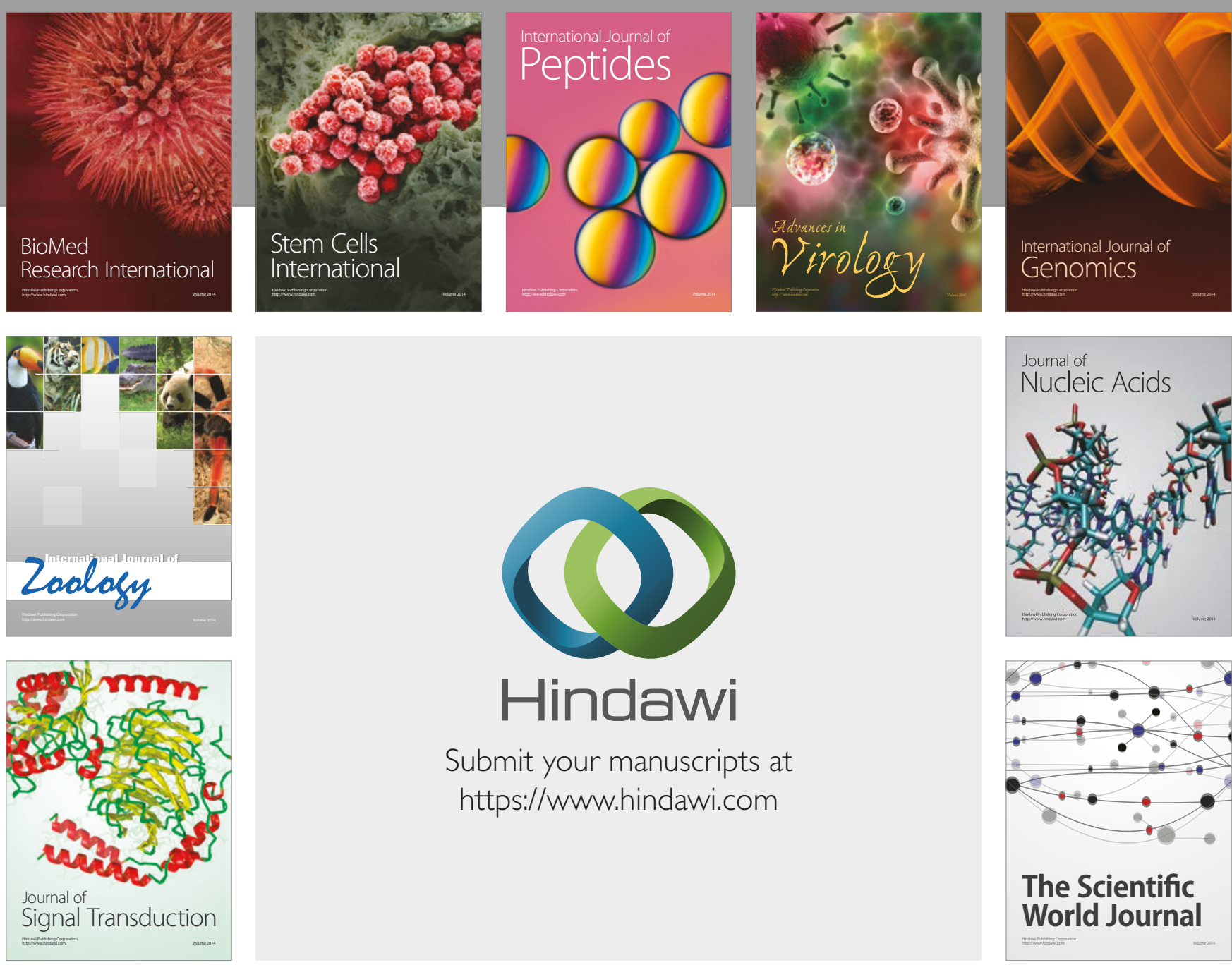

Submit your manuscripts at

https://www.hindawi.com
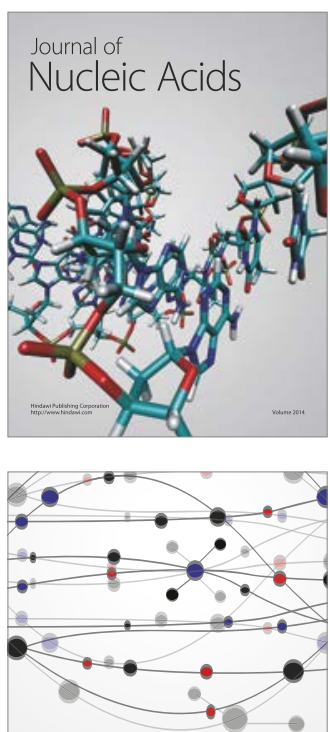

The Scientific World Journal

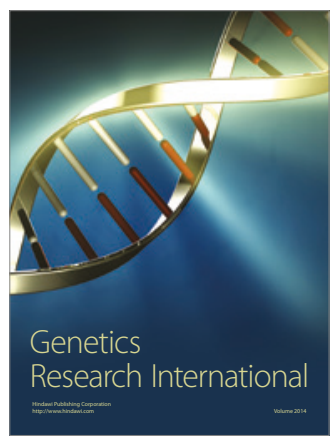

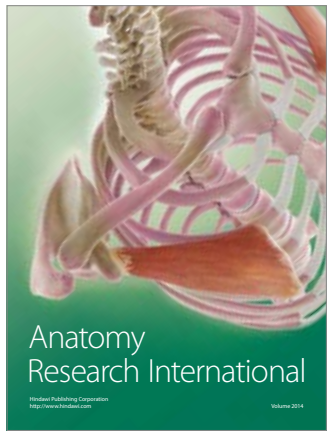

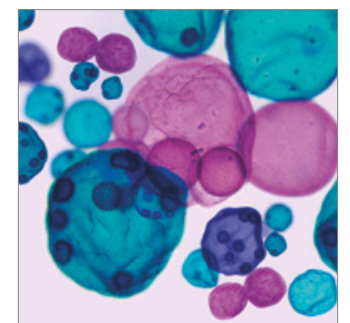

International Journal of Microbiology
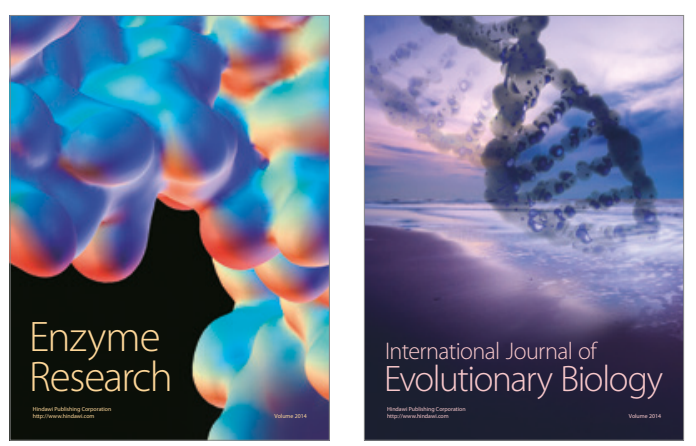
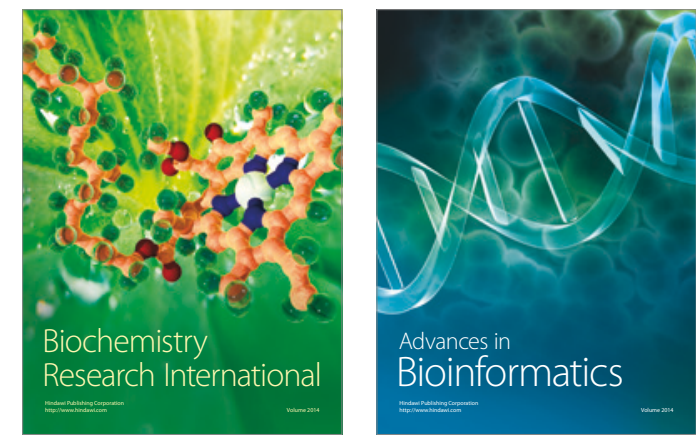

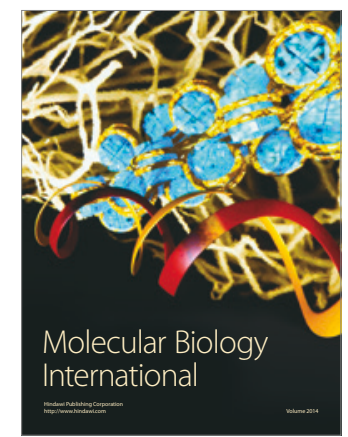

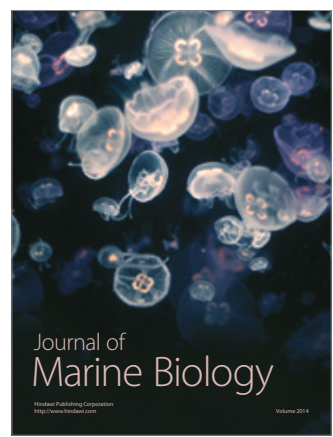

